\title{
M-ARRAY QUADRATURE AMPLITUDE MODULATION WIRELESS SENSOR NETWORK MODULATOR RELIABILITY AND ACCURACY ANALYZE IN CIVIL SHM
}

\author{
${ }^{1}$ Mohammud Ershadul Haque, ${ }^{1}$ Mohammud Fauzi Mohammud Zain, \\ ${ }^{1}$ Maslina Jamil, ${ }^{1}$ Mohammud Abdul Hannan and ${ }^{2}$ Abdulla Al Suman \\ ${ }^{1}$ Faculty of Environment and Built Engineering, Universiti Kebangsaan Malaysia (UKM), Malaysia \\ ${ }^{2}$ Department of Electronics and Telecommunication Engineering, \\ Rajshahi University of Engineering and Technology (RUET), Bangladesh
}

Received 2013-07-02, Revised 2013-10-10; Accepted 2013-10-29

\begin{abstract}
Wireless Sensor Network (WSN) is the new invention applying for assessment the damage of the historical or high rise civil building structural health. Technical challenges affecting deployment of wireless sensor network including the range of the transmission problem, low data transmission rate of the existing SHM strategies. The most vital factor of SHM wireless sensor systems is the modulator accuracy and reliability that qualify the wireless communication system to assess large building structure health Information. The objective of this article is to provide solution to measure both reliability and accuracy of the wireless sensor network modulator. we computed M-array QAM modulator BER and compare the simulation result with theoretical to find out optimum modulation technique for transmission System with considering maximum data rate, AWGN channel and also measured modulator accuracy based on ZigBee by computing M-array modulator Error Vector Magnitude (EVM) to quantify the transmitter quality.
\end{abstract}

Keywords: WSN, SHM, BER, EVM, Reliability and Accuracy

\section{INTRODUCTION}

Now a day, safety issue related to the high-rise building or historical building is most important to the human society. The health of the civil building structural performing a key task in evaluate consistency of a structure and identify possible damaging factor that are fundamental element to define the danger level of a building. Currently, wired based sensing measurement technique is being employed to obtain civil building structural health sensing information. In the patient area, a typical sensing system consists of a sensing system, data collection system and computing system. To measure the whole patient area many sensor device connected with base station through wired based data collection system. Single hop wireless communication system is exercised in the lighter amount of structural health monitoring system.
There are several disadvantages are found in the monitoring system among those adding extra sensor node, transmitter power communication range. Due to technology develop Wireless Sensor Network (WSN) technology solve some drawback not all related to the whole system in recent years. Technology develops in ZigBee device open numerous application of the WSN. Each sensor node in the monitoring system sense structural response and transmit its sensing data over the air in the case of wireless and received signal are analyzed at the computing section to discover how much damage occur in the building structure (Jianjun et al., 2009). The embedded sensor system provides many sensors to measure the structural system parameter easily and whole system becoming low cost, low power profile. The parameter measure of the structural health response depends of the sensing parameter of the sensor. Structural

Corresponding Author: Mohammud Ershadul Haque, Faculty of Environment and Built Engineering,

Universiti Kebangsaan Malaysia (UKM), Malaysia 
health damage extremely occurs cause to natural events like seismic, extreme event or heavily loaded on structure. The remaining life time of the structure depend on how much affect by the attack of structural diseases like deterioration, corrosion, fatigue, vibration. So, lower cost monitoring system is requisite to determine the safety level about structural degradation. Visual inspection of the structural damage discovers the perceptible injure on the structural surface. To perceive structural diseases and timely report to the base station a real time monitoring system requires that autonomously and pro-actively monitor the structural health. The definition of the structural health monitoring system can be given that way it's the process or tactics of the any kind of structural health damage assessment tool. That harm defined by the material change or geometrical property change of the structure (Farrar and Worden, 2007). The final goal of the of the structural health monitoring system is safety level monitoring of the structural clash with lighter system cost Wired based data collection system is the traditional system that collect sensor data periodically (Haque et al., 2012). Non-Destructive Inspection (NDI) method has been used from many years in event agenda, preservation of the building structural health monitoring system. In this technique, hardware implementation, simulation procedure, statistical tactic, signal processing algorithm considerably improvement (Achenbach, 2009). Structural health monitoring system concerns with specific field of any kinds of structure the covenant the scratch detection, solidity and uprightness of infrastructure. Among many kinds of structure, civil infrastructure like high-rise buildings and bridges are a fundamental element of our social life. The infrastructure of our social development especially high-rise building and long bridge more complex so a reliable, automated and robust control monitoring system is emergently necessary that pin point the structural health damage. Typically, the detection of the damage of the large structure figure out physically by specialist with defines parameter. But, this physical assessment to high cost, unable to determine damage frequency and more possibility of the error in the assessment result. The accuracy of the result depends on the precision and accuracy of the measuring instrument. Digital form of acquire data from sensing device provides the error due to the data Analog to Digital Converter (ADC) conversion (Lo Re and Ortolani, 2009).

\section{BACKGROUND AND MOTIVATION}

Corrosion is a universal problem of structural failure. Already many sensors developed to measure the corrosion of the structure those are able to predict remain durability and maintenance of the structure. Ultrasonic sensor is used to measure the corrosion but high installation is the shortcoming of this sensor. Not only, had the lifetime of this sensor system much shorter and more power required. So, optimum design of the ultrasonic is too necessary to reduce the system cost (Jin and $\mathrm{Wu}, 2010)$. After adding the low microcontroller to the sensor system becoming a smart system as popular term. This smart sensor system replaces the traditional analog sensor and provided digital sensors. These smart sensors offer low cost, low power profile improve SNR ratio and easy to interface with the network element. The smart sensor is easily applicable in environment to measure various parameters to provide automated environment system (Flammini et al., 2009). The concrete structural health evaluation, test and administration task done by sensor network monitoring system. The long term structural health monitoring already developed with fifty sensor node that are applicable into the practical field. Currently, rising number of research study on sensor technology develop in civil structural health from important parameter ecological weather. Using fiber optic technique a number of cracks can be sensed, positioned and observed. There are several number of predetermined acknowledgment require about damage location and able to measure various types of parameter of the different structure. Warmth and humidity of the structural health measure has been recently proposed in (Barroca et al., 2013). This measurement method use MEMS sensor to measure temperature and moisture with micro-cantilever. Due to advance technology development in wireless smart sensors applications open numerous opportunities to measure high-rise civil building structural health measure (Wang et al., 2007; Gao et al., 2006; Nagayama et al., 2008). Still have few limitation of wireless sensor network, remarkably less reliable data transmission than wired systems, a comparatively short communication range, however, many studies showed that Wireless smart sensors can be used to make a reliable and accurate structural health monitoring system (Weng et al., 2008; Lynch et al., 2004; 2006). There are many factors that affect the communication system in practical environments and Many Researcher shows that employed with Wireless Sensor Network (WSN) numerous problem arises among those in terms of communication system (Haque et al., 2013a). A star topology based sensor network loss is computed by Haque et al. (2013b) and provide the optimum buffer mechanism technique. 


\subsection{Objective}

The major objective of this multidesiplinary research is to compute M-array BER, EVM for Wireless Sensor Network allowing for tenuously monitor certain civil structural health. Generally, WSN are consisting of senor, micro-controller and communication module. Those sensors are able to sense real event environment and transmit the sensor information to the base-station through wireless transmission. So reliable communication system is the vital issue to send the respective base station without any error. The modulator reliability and accuracy one of the major concern to received signal at the destination correctly. This is accomplished by Computing Bit Error Rate (BER), Error Vector Measurement (EVM) based on ZigBee enabling the significant improvement of system reliability and accuracy. In the application of the WSNs practical field to structural health engineering is significant to produce monitoring product platform that is capable to contain a broad range of sensor system, based on customer demand exchange information between node and gateway/base station without information loss occur at the transmitter or receiver. The aim of this article the output from this research is to qualify the modulator accuracy of the wireless sensor network.

\section{SYSTEM AND APPROACH DESCRIPTION}

The goal of this study is to analyze M-array QAM modulator accuracy and to validate the modulator performance to offer optimum sensor network performance to provides the higher data rate wireless sensor network in civil structural health monitoring. The analysis system consist of two nodes one transmit sensor node and another is received sensor node or base station.

\subsection{QAM Modulator}

QAM is an admired modulation technique that is frequently used in the wireless communication system. ASK and PSK modulation techniques are used to form QAM modulator. QAM presented by four quadrants and this four quadrant contain ASK and PSK modulated signal. It is possible to send M-QAM modulation signal using ASK and PSK four quadratic method. Below figure shows the formation of the QAM modulator scheme. A binary data series $d(t)$ consist as input of 2bit serial to parallel converter with $\mathrm{R}$ bps data rate. Then, 2-bit serial to parallel converter produce two level of $\mathrm{R} / 2$ data rate bit stream. At the upper level of the modulation technique, outgoing data rate from serial to parallel converter multiplying by the career signal generated by oscillator. In the upper scheme binary one represent by career signal with constant amplitude and zero represent by absence of carrier signal. At the lower portion of the modulation technique, firstly carrier signal phase are shifted by $90^{\circ}$ and multiplying this shifted signal by the lower bit stream data rate. After than both multiplied signal are added together and transmit this signal as a QAM modulated signal. The following equation is used to represent the transmitted QAM signal Equation 1:

$s(t)=d_{1}(t) \cos 2 \pi f_{c} t+d_{2}(t) \sin 2 \pi f_{c} t$

The relation between the Level (L) and required number of bits ( $\mathrm{n}$ ) is denoted by the following relationship Equation 2:

$\mathrm{L}=2^{\mathrm{n}}$

The below shows the modulator and demodulator architecture. At the QAM demodulator section, the inverse operation of the modulator is taken place and below Fig. 1 and 2 shows the QAM demodulator architecture.

\subsection{BER Simulation Method and System}

The performance of the wireless sensor network is determined by BER of the transmission system. Its define how much bits change its state during transmission from transmitter to receiver. This change may be due to channel noise, interference, distortion or bit synchronization error. It can be express by the following manner Equation 3:

$$
\text { BER }=\frac{\text { Total number of Error Bits During Transmission }}{\text { Total Number of Transmitted Bits }}
$$

It determine the reliability of the enter radio transmission system and it represent as fractional value. The value of the BER may be varying by changing the modulation scheme in the system. In the presence of impairment of transmission through electronic circuitry and the propagation medium BER become significant. A detail description of the BER is found in. To compute the performance of the WSN for monitoring building structural health the following Fig. 3 is considered for computing BER. 


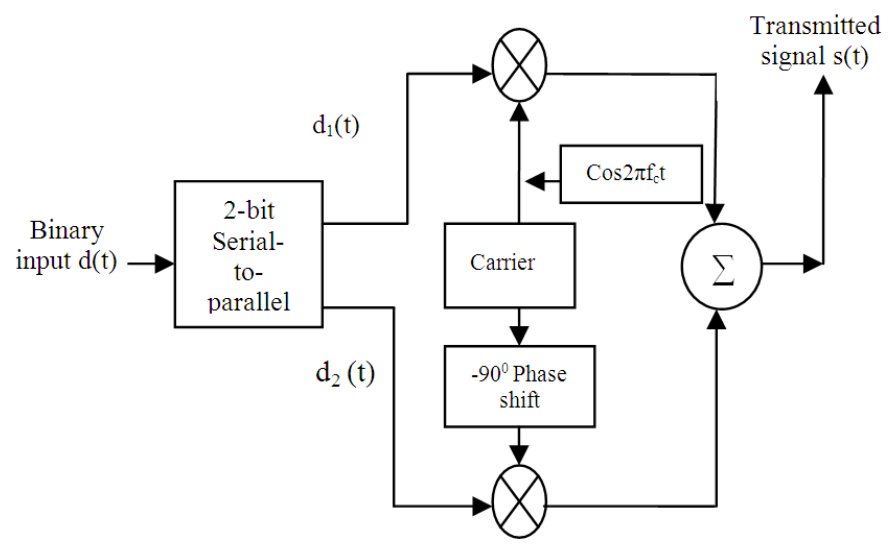

Fig. 1. QAM-modulator architecture

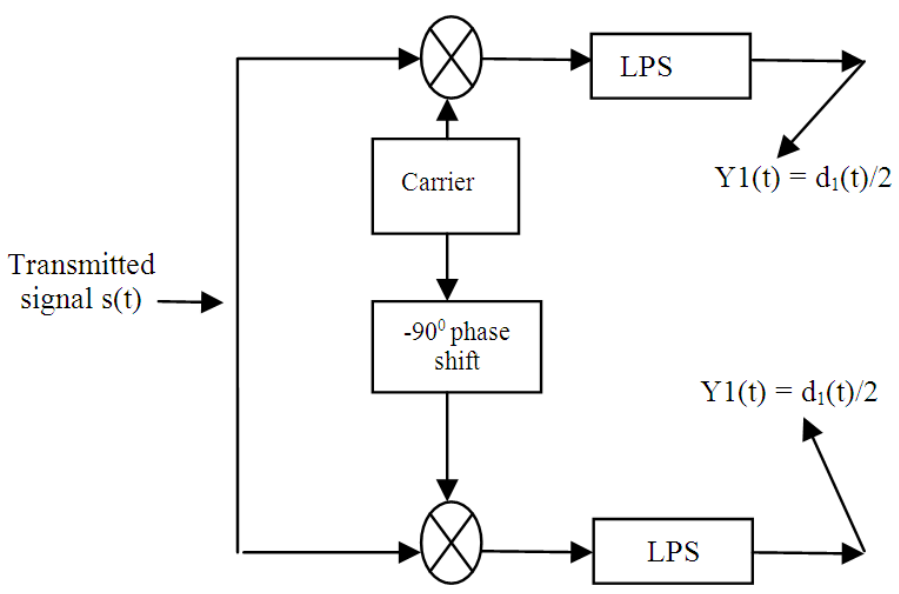

Fig. 2. QAM-demodulator architecture

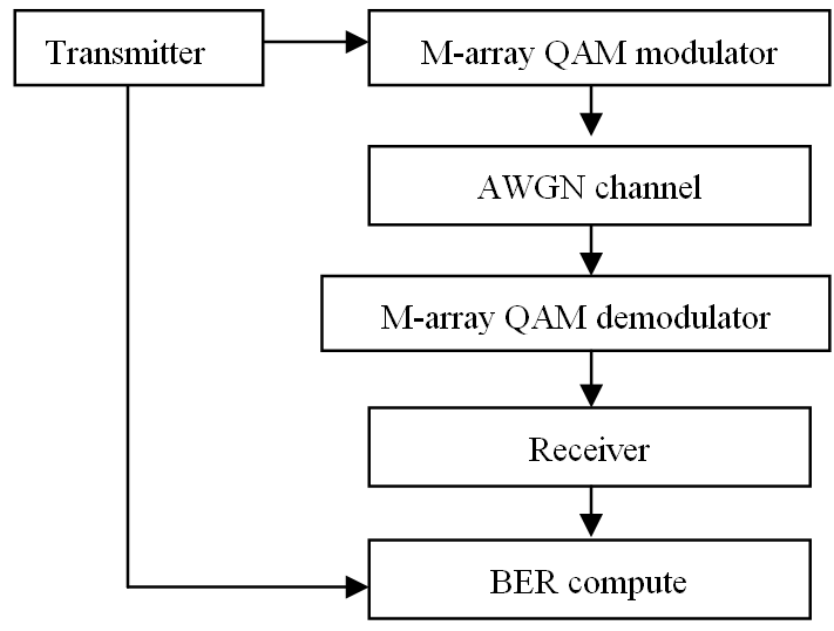

Fig. 3. M-array QAM BER computing mechanism 


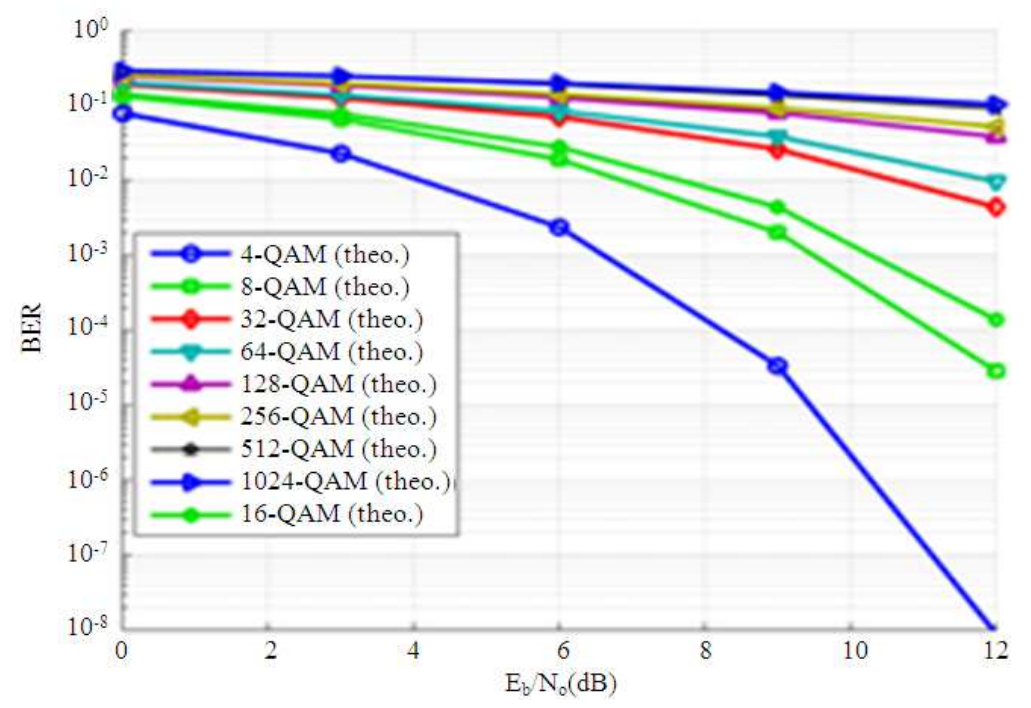

Fig. 4. M-array QAM Modulator BER theoretical

Transmitter generate the random signals consist of the M-array data and transmit the signal to the QAM modulator. Modulator modulates the career signal according to the incoming message. It also modulate the signal into two component one is in-phase and another is quadrature component which is consist of ASK and PSK. After modulated, the modulating signal is passes through Additive White Gaussian Noise (AWGN) which add noise with modulated signal. After adding noise, noisy signal is demodulated using QAM demodulator and finally received signal at receiver. BER compute part calculate BER by comparing the received signal at the receiver with the transmit signal from transmitter.

The BER simulation and theoretical result for $\mathrm{M}$ array QAM are given above Fig. 4.

Figure 4 depict the theoretical result for M-array QAM. This figure shows that as increasing value of the modulator order, the BER value is going to higher that means lower order QAM modulator provides better performance than higher order QAM modulator. Figure 5 represents the M-array QAM BER simulation result. Simulation result shows the same result as theoretical but in the case of higher order like 128, 256, 512 and 1024 the result is not same as theoretical some difference result are observed for BER result.

\subsection{Error Vector Measurement (EVM) Analysis}

In wireless communication system, transmitted signal phase and amplitude is distorted created by the power amplifier directly affect the quality of the transmitted signal.
In the latest communication system protocols, the Error Vector Magnitude (EVM) is the most significant measurement for analyzing power amplifier performance.EVM determine the modulator performance, or how much error free signal is modulated, represented by the transmit signal phase and amplitude. Insight into the communication link, transmit signal performance is measured by evaluating EVM value.

\subsubsection{System Model}

Firstly, we generate chip value to implement DSSS technique. After than, mapping the spreading gain with assume data rate for implementing chip rate. Then, we design pulse shaping raised cosine filter with considering design parameter and set roll of factor to 1. After implementing symbol its pass through pulse shaping raised cosine filter. The EVM object assume that reference signal and received are synchronized and sample at same rate and compute EVM value base on this. The computational EVM measurement results for M-array QAM modulators are given below.

From Table 1 at the SNR $=12 \mathrm{db}$, the minimum worth case RMS EVM value recorded at 4-QAM and maximum at 128-QAM and the other value of EVM increasing as increasing the modulation order without percentile EVM. At lower value of the EVM measurement parameter provided better modulator accuracy and minimum value of the worth case RSM EVM $30 \%$ defined by the (IEEE Standard 802.15.4). Table $\mathbf{2 - 5}$ illustrate the computational result of the M-array QAM modulator for $\mathrm{SNR}=24,36,48$ and 60 respectively. 


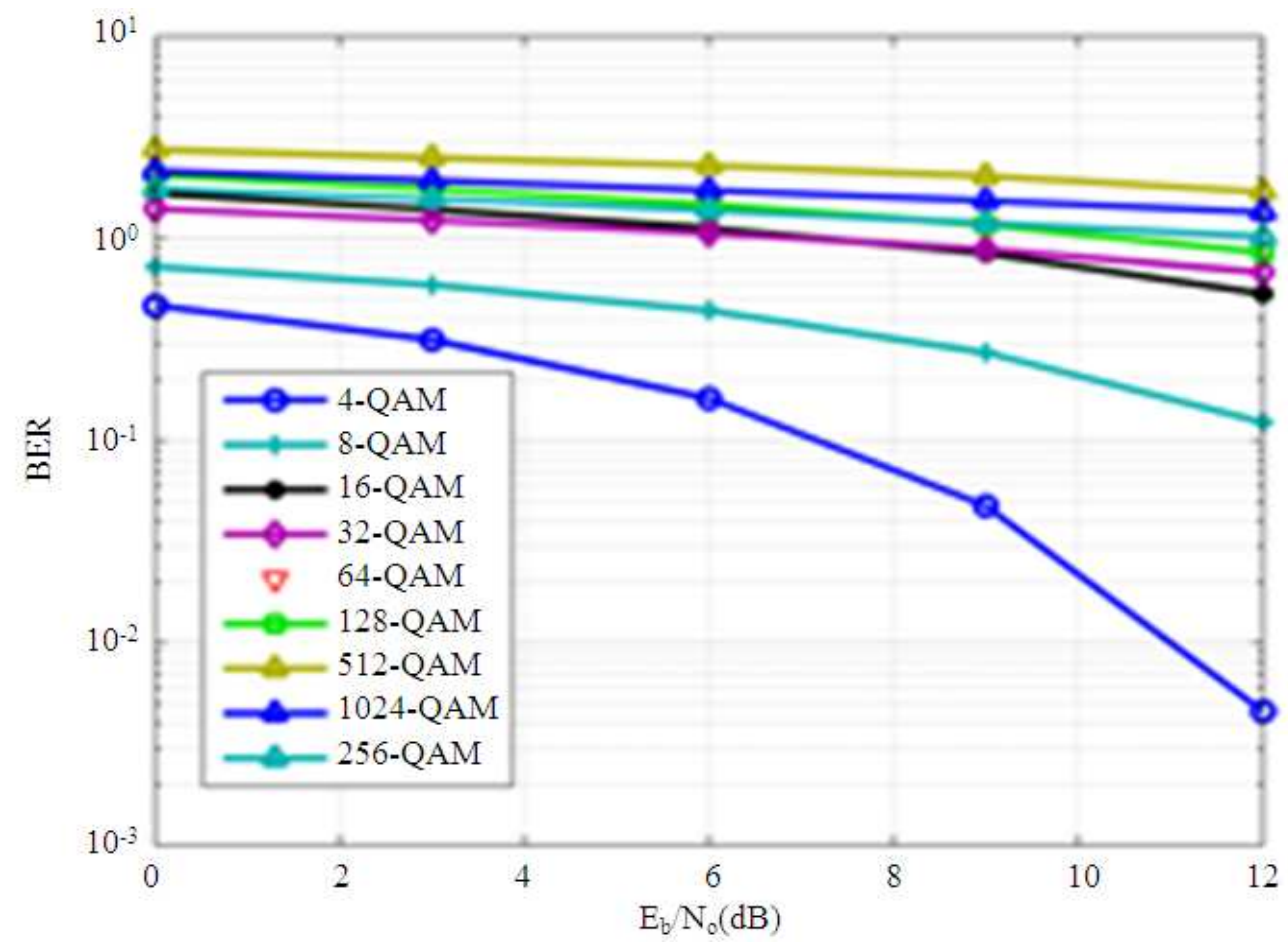

Fig. 5. M-array QAM Modulator BER Simulation

Table 1. $\mathrm{SNR}=12$. Peak power $=1$, Number of symbols $=120000000$

\begin{tabular}{llllc}
\hline Modulator & RMSEVM & Maximum EVM & Percentile EVM & Worst case RMS EVM $(\%)$ \\
\hline 4-QAM & 193.0863 & 897.8555 & 327.3127 & 193.11 \\
8-QAM & 376.1221 & $1.66 \mathrm{E}+03$ & N/A & 376.27 \\
16-QAM & 809.1224 & $3.57 \mathrm{E}+03$ & N/A & 809.56 \\
32-QAM & $1.98 \mathrm{E}+03$ & $8.95 \mathrm{E}+03$ & N/A & 1984.33 \\
64-QAM & $4.04 \mathrm{E}+03$ & $1.72 \mathrm{E}+04$ & N/A & 4039.51 \\
128-QAM & $8.15 \mathrm{E}+03$ & $3.68 \mathrm{E}+04$ & N/A & 8154.40 \\
\hline
\end{tabular}

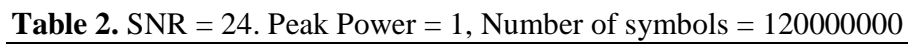

\begin{tabular}{llllr}
\hline Modulator & RMS EVM & Maximum EVM & Percentile EVM & Worst case RMS EVM $(\%)$ \\
\hline 4-QA & 48.5018 & 209.0612 & 83.9511 & 48.52 \\
94.4922 & 94.4922 & 426.581 & 163.5425 & 94.58 \\
16-QAM & 203.2454 & $1.01 \mathrm{E}+03$ & 341.0248 & 203.40 \\
32-QAM & 498.2429 & $2.13 \mathrm{E}+03$ & N/A & 498.42 \\
64-QAM & $1.01 \mathrm{E}+03$ & $4.41 \mathrm{E}+03$ & N/A & 1015.44 \\
128-QAM & $2.05 \mathrm{E}+03$ & $8.73 \mathrm{E}+03$ & N/A & 2048.22 \\
\hline
\end{tabular}

Table 3. $\mathrm{SNR}=36$. Peak Power $=1$, Number of Symbols $=120000000$

\begin{tabular}{lcccc}
\hline Modulator & RMSEVM & Maximum EVM & Percentile EVM & Worst case RMS EVM $(\%)$ \\
\hline 4-QAM & 12.1823 & 52.5920 & 21.0839 & 12.19 \\
8-QAM & 23.7373 & 101.2930 & 41.0811 & 23.75 \\
16-QAM & 51.0563 & 228.4335 & 88.3652 & 51.06 \\
32-QAM & 125.1474 & 559.3549 & 216.5896 & 125.17 \\
64-QAM & 254.8338 & $1.14 \mathrm{E}+03$ & 395.0331 & 254.85 \\
128-QAM & 514.2683 & $2.32 \mathrm{E}+03$ & N/A & 514.51 \\
\hline
\end{tabular}


Table 4. $\mathrm{SNR}=48$. Peak Power $=1$, Number of Symbols $=120000000$

\begin{tabular}{lcccc}
\hline Modulator & RMS EVM & Maximum EVM & Percentile EVM & Worst case RMS EVM (\%) \\
\hline 4-QAM & 3.0601 & 13.1851 & 5.2964 & 3.0700 \\
8-QAM & 5.9619 & 26.3442 & 10.3191 & 5.9600 \\
16-QAM & 12.8258 & 55.8407 & 22.1979 & 12.8400 \\
32-QAM & 31.4348 & 141.8714 & 54.4059 & 31.4500 \\
64-QAM & 64.0105 & 317.9557 & 110.7825 & 64.0400 \\
128-QAM & 129.1784 & 583.0065 & 223.5515 & 129.2400 \\
\hline
\end{tabular}

Table 5. $\mathrm{SNR}=60$. Peak power $=1$, Number of symbols $=120000000$

\begin{tabular}{lcccc}
\hline Modulator & RMS EVM & Maximum EVM & Percentile EVM & Worst case RMS EVM $(\%)$ \\
\hline 4-QAM & 0.7687 & 3.2392 & 1.3305 & 0.77 \\
8-QAM & 1.4975 & 6.3197 & 2.5918 & 1.50 \\
16-QAM & 3.2216 & 13.7058 & 5.5758 & 3.22 \\
32-QAM & 7.8966 & 33.6882 & 13.6664 & 7.90 \\
64-QAM & 16.0778 & 70.9765 & 27.8264 & 16.09 \\
128-QAM & 32.4504 & 138.4383 & 56.1604 & 32.46 \\
\hline
\end{tabular}

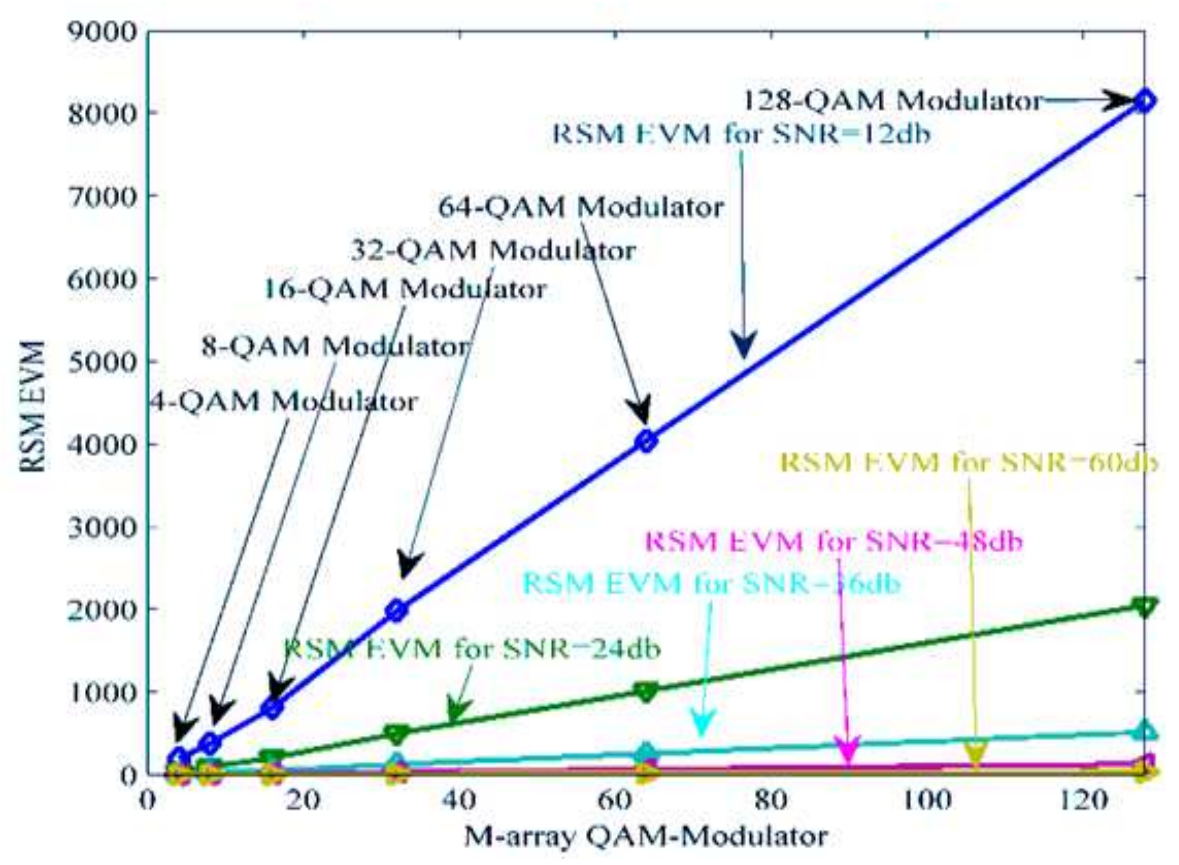

Fig. 6. RMS EVM of M-array QAM-Modulator

From all computational result, we can told that an increasing value of the modulator level EVM parameter value going to decrease with increasing value of the SNR. The below Fig. 6-8 represent the RSM EVM, Maximum EVM and worth case EVM for different level of SNR value.

Figure 6 demonstrations that as increasing value of the modulation order with SNR lead the increasing value of RSM EVM and minimum value recoded at 4QAM at $60 \mathrm{db}$ SNR.
On the other hand Fig. 7 describe the Maximum EVM value for M-array QAM modulator. The maximum EVM value recorded at 4-QAM modulator for $12 \mathrm{db}$ SNR and minima recorded at 4-QAM for 60 db SNR.

Lastly, the minimum worst case value obtained at 4QAM for $60 \mathrm{db}$ SNR and minimum worth case condition exceed at 128-QAM modulator for $60 \mathrm{db}$ SNR value. The below Fig. 8 illustrate the worst case value that determine the modulator accuracy. 


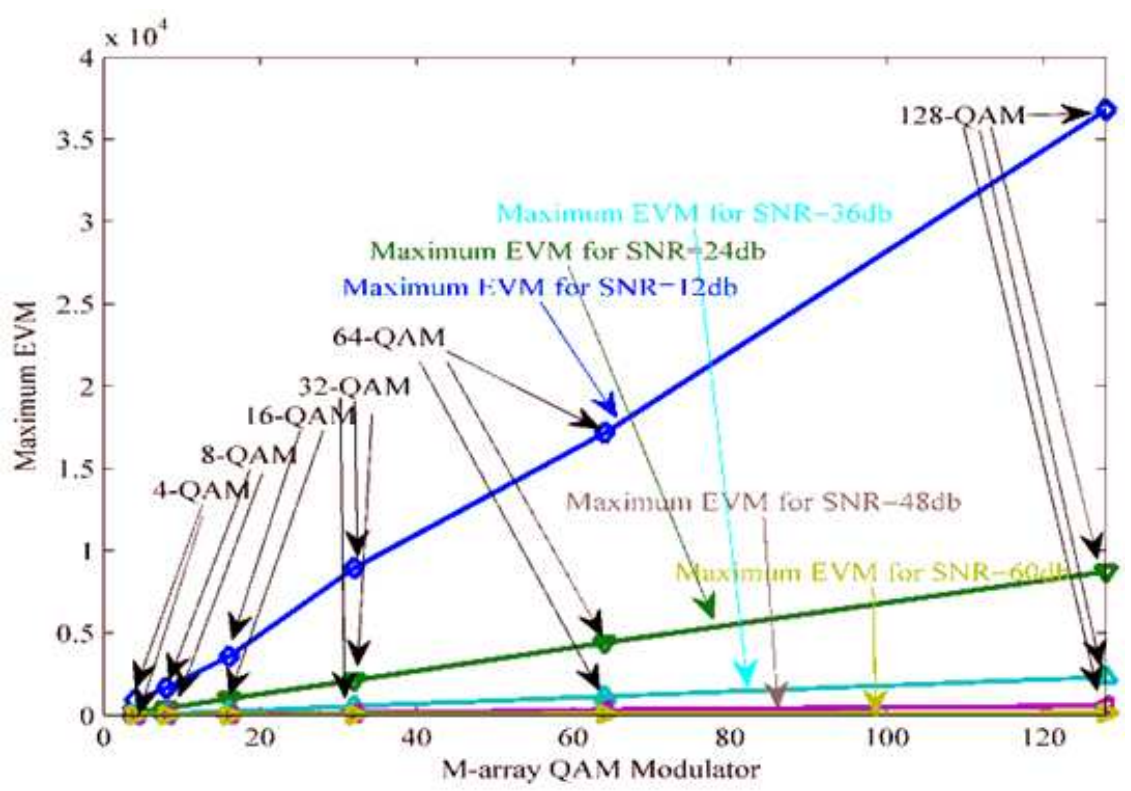

Fig. 7. Maximum EVM of M-array QAM-Modulator

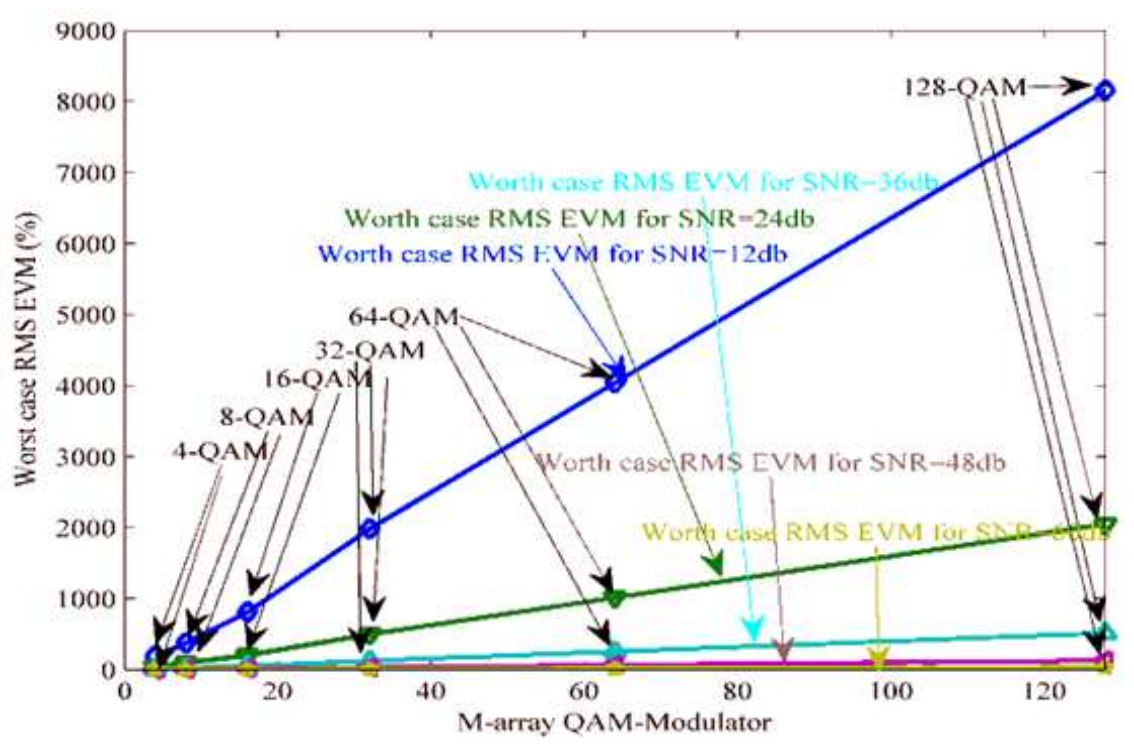

Fig. 8. Worth case RMS EVM of M-array QAM Modulator

\section{CONCLUSION}

In this article, we investigated the greater realiability and accuracy of the wireless sensor network modultor. From above investigation we can told that, the analytical result shows that as as increasing the QAM modulator order, the worth case performance are seen in the case of higher order modulator than lower order QAM modulator. But, simulation shows that there is some different result observe in which sometimes higher order QAM modulator exibit better performance than lower order. For reliability, generally we conclude that lower order QAM modullator behave as better reliability than higher order. On the other hand, for accuracy 
measurement, higher order modulator offers greater accuracy than lower order. At standared level is obtained for 128-QAM modulator with $60 \mathrm{dBm}$ of SNR value. From bove investigation, there are two contradictory think related to each other one is the BER and another is EVM. If one is increase than another one is decrease. The modulator realiability and accuracy define by the desighner interest and his major application planing part priority.

\section{ACKNOWLEDGEMENT}

WISUDA SDN. BHD. gratefully acknowledges for supporting this Research Program.

\section{REFERENCES}

Achenbach, J.D., 2009. Structural health monitoringWhat is the prescription? Mech. Res. Commun., 36: 137-142.

Barroca, N., L.M. Borges, F.J. Veleza, M. Górski and J. Castro-Gomesb, 2013. Wireless sensor networks for temperature and humidity monitoring within concrete structures. Construct. Build. Mater., 40: 1156-1166.

Farrar, C.R. and K. Worden, 2007. An introduction to structural health monitoring. Phil. Trans. Roy. Soc., 365: 303-315. DOI: 10.1098/rsta.2006.1928

Flammini, A., P. Ferrari, D. Marioli, E. Sisinni and A. Taroni. 2009. Wired and wireless sensor networks for industrial applications. Microelectronics J., 40: 1322-1336. DOI: 10.1016/j.mejo.2008.08.012

Gao, Y., B.F. Spencer and M. Ruiz-Sandoval, 2006. Distributed computing strategy for structural health monitoring. Struct. Control Health Monit., 13: 488507. DOI: $10.1002 /$ stc. 117

Haque, M.E., M.F.M. Zain, M.A. Hannan, M. Jamil and H. Johari, 2012. Recent application of structural civil health monitoring using WSN and FBG. World Applied Sci. J., 20: 585-590.

Haque, M.E., M.F.M. Zain, M.A. Hannan, M. Jamil and H. Johari, 2013a. Loss monitoring of star topology sensor network based on scheduling algorithm for assessing structural health information. Am. J. Applied Sci., 10: 1484-1491. DOI: 10.3844/ajassp.2013.1484.1491
Haque, M.E., M.F.M. Zain, M.A. Hannan, M. Jamil and M.H. Johari, 2013b. Transmission loss computed of star topology sensor network base on DT, RED and SFQ buffer mechanism for overseeing high rise building structural health. Int. J. Biosci., Biochem. Bioinform., 3: 646-649. DOI: 10.7763/IJBBB.2013.V3.294

Jianjun, N., D. Zhidong, F. Deng Zhou and S.C. Zong, 2009. A structural health monitoring system using wireless sensor network. Proceedings of the 5th International Conference Communications, Networking and Mobile Computing, Sept. 24-26, IEEE Xplore Press, Beijing, pp: 1-4. DOI: 10.1109/WICOM.2009.5302988

Jin, W. and W. Wu, 2010. Study on wireless sensing for monitoring the corrosion of reinforcement in concrete structures. Measurement, 43: 375-380. DOI: 10.1016/j.measurement.2009.12.003

Lo Re, A.G.G. and M. Ortolani, 2009. WSNs for structural health monitoring of historical buildings. Proceedings of the 2nd Conference Human System Interactions, May 21-23, IEEE Xplore Press, Catania, pp: 574-579. DOI: 10.1109/HSI.2009.5091041

Lynch, J.P., K.H. Law, A.S. Kiremidjian and E.D. Carryer, 2004. Design and performance validation of a wireless sensing unit for structural monitoring applications. Structural Eng. Mech., 17: 3-4.

Lynch, J.P., Y. Wang, K.J. Loh, J.H. Yi and C.B. Yun, 2006. Performance monitoring of the Geumdang Bridge using a dense network of high-resolution wireless sensors. Smart Mater. Struct., 15: 15611561. DOI: 10.1088/0964-1726/15/6/008

Nagayama, T., B.F. Spencer, K. Mechitov and G. Agha, 2008. Middleware services for structural health monitoring using smart sensors. Smart Structures Syst.

Wang, Y.A., P.J. Lynch and H.L. Kincho, 2007. A wireless structural health monitoring system with multithreaded sensing devices: Design and validation. Struct. Infrastructure Eng.: Maintenance, Manage. Life-Cycle Design Perform., 3: 103-120. DOI: 10.1080/15732470600590820

Weng, J.H., C.H. Loh, J.P. Lynch, K.C. Lu and P. Lin et al., 2008. Output-only modal identification of a cable-stayed bridge using wireless monitoring systems. Eng. Struct., 30: 1820-1830. DOI: 10.1016/j.engstruct.2007.12.002 